\title{
Awareness on Risk Factors Associated with Oral Cancer Among School Students in Chennai
}

\section{Sushma B1, Vishnu Priya V², Karthik Ganesh Mohanraj³, Gayathri R}

Section: Healthcare

Sci. Journal Impact

Factor: 6.1 (2018)

ICV: 90.90 (2018)

(c) (3) (8)

Copyright@IJCRR

\author{
'Saveetha Dental College, Saveetha Institute of Medical and Technical Sciences (SIMATS), Saveetha University, Chennai, India; ${ }^{2}$ Department of \\ Biochemistry, Saveetha Dental College, Saveetha Institute of Medical and Technical Sciences (SIMATS), Saveetha University, Chennai, India; \\ ${ }^{3}$ Department of Anatomy ,Saveetha Dental College,Saveetha Institute of Medical and Technical Sciences (SIMATS),Saveetha University, Chen- \\ nai, India; ${ }^{2}$ Department of Biochemistry, Saveetha Dental College, Saveetha Institute of Medical and Technical Sciences (SIMATS), Saveetha \\ University, Chennai, India.
}

\section{ABSTRACT}

Introduction: Oral cancer is also known as mouth cancer that is the cancer of the lining of the lips, mouth, or upper throat. In the mouth, it commonly starts as a painless white patch and develops into red patches. It commonly looks like a persistent ulcer that does not heal and slowly grows. Symptoms may include difficult or painful swallowing, new lumps in the neck, swelling in the mouth, feeling of numbness in the mouth, or lips and risk factors include tobacco and alcohol use.

Objective: To create awareness of risk factors of oral cancer among school students.

Methods: The study setting was done using a prospective observational study in Saveetha Dental College (SDC). Approval is received from the scientific review board, (SDC). The sampling method is random sampling including the sample size of 100 participants. A self-structured questionnaire was prepared including 15 questions.

Results: The results were collected and analyzed using statistical analysis using online SPSS software. According to the results, $65 \%$ of school students are aware of oral cancer. Almost $70 \%$ of school students believe that oral cancer leads to death. Students also know that smoking causes oral cancer.

Conclusion: The survey demonstrated that school students are aware of risk factors associated with oral cancer but there is a lack of knowledge about it. So, creating awareness on knowledge of risk factors of oral cancer will be helpful for future generations.

Key Words: Awareness, Oral cancer, Oral hygiene, Risk factors, Smoking, Risk factors, Tobacco, Online survey

\section{INTRODUCTION}

Oral and pharyngeal cancers can be prevented and successfully treated when diagnosed at an early stage. Lack of knowledge of practice among dentists has shown to contribute to delayed treatment. Early detection of oral cancers makes them more amenable to treatment and allows the early cure. ${ }^{1}$ Almost $90 \%$ of oral cancers are squamous cell carcinomas. Smoking, drinking alcohol, and HPV infections are the major risk factors, with a high risk of oral cancer. Despite the recent advances in detection and treatment of cancer, visual accessibility of the oral mucosa, and the scientific knowledge on oral cancer and their risk factors showed a low survival rate (nearly $50 \%)^{2}$

But in most countries, the incidence of oral cancer is relatively low when compared with other malignancies, it is considered a major public health issue due to low five-year survival rates. These rates are correlated to a high extent to the often-advanced stage of the disease during diagnosis and treatment. Van der Waal has reported a 5-year survival rate for oral cancer stage- 1 at approximately $80 \%$, while the corresponding value for patients with advanced disease (StageIII/ Stage-IV) is almost $20 \%{ }^{3}$

Looking into the history, the level of risk factor knowledgeability surrounding oral cancer has been markedly low, with only $1 / 4$ th of individuals recognizing tobacco as an OC-risk factor. However, knowledge on tobacco exposure as a dominant risk factor for oral and other cancers is now highlighted, so the relationship between alcohol misuse and OC remains under-appreciated. ${ }^{4}$ It has been proved that lack of awareness among the public about oral cancer and the associated risk factors is the first and foremost reason for the late presenta-

\section{Corresponding Author:}

Vishnu Priya V, Professor, Department of Biochemistry, Saveetha Dental College, Saveetha Institute of Medical and Technical Sciences (SIMATS), Saveetha University, Chennai, India; Email: vishnupriya@saveetha.com

ISSN: 2231-2196 (Print)

Received: 16.09 .2020
ISSN: 0975-5241 (Online)

Revised: 17.10 .2020
Accepted: 14.11 .2020
Published: 26.12 .2020 
tion of oral cancer. The objective of the present study was to determine the level of awareness of oral cancer and the associated risk factors in the high-risk population of Kerala, India. This knowledge is used to implement an effective health education program to reduce the incidence of oral cancer. ${ }^{5}$

Lack of awareness of the risk factors for oral cancer leads to late presentation of the disease which contributes to the poor survival of patients who contract it. Intra-oral cancer has a high incidence in the northeast of England. ${ }^{6}$ Public awareness of oral cancer was poor but there is some confirmation that certain groups may be more aware. More people may have heard of mouth cancer thanks to initiatives like Mouth Cancer Awareness Week (MCAW) or the West of Scotland Cancer Awareness Project (WoSCAP). ${ }^{7}$

Almost all the previous articles focused on older ages and few articles had done surveys among both school and college students regarding oral cancer. But the understanding of risk factors of oral cancer is very essential in the case of schoolaged children. Previous studies on enzyme assays, obesity, ${ }^{8}$ cirrhosis, nanomaterials, cancer biology, and natural compounds. ${ }^{9-22}$ enriched the knowledge and this made me do an epidemiological study that is needed for the community. So, the aim of this study is to create awareness of risk factors of oral cancer among school students.

\section{MATERIALS AND METHODS}

The study setting was done using a prospective observational study. Approval for the study is received from the scientific review board. The study included a sample size of 100 school students using the non-probability convenient sampling method. Measures are taken to minimize errors in the questions and avoid leading questions. A self-structured questionnaire was prepared including 15 questions and circulated among the 100 school students using an online Google forms link. The method of representation of each output is pie-chart representation. The statistical test used is descriptive statistics using SPSS software, estimating the independent variables like height, weight, risk factors of other disease, and dependent variables like awareness, interaction, knowledge, attitude, and perception.

\section{RESULTS AND DISCUSSION}

$65 \%$ of school students were aware of oral cancer (Figure 1a). Almost $70 \%$ of the school students believe that oral cancer leads to death (Figure 2a) and even $68.37 \%$ of students were taught about oral cancer and its complications in their school (Figure 3a). $60 \%$ of the students are able to know the symptoms of oral cancer. So, it can be cured fast (Figure 4a).

$68.69 \%$ of the school students were aware that oral cancer can be cured using chemotherapy and radiotherapy. This might be due to some of their parents being doctors (Figure $5 a)$. Students strictly know that smoking can cause oral cancer (Figure 6a). $55.56 \%$ of people believe that oral cancer can be cured through proper diagnosis even if the patient is from a rural area (Figure 7a).

Only $59 \%$ of the students are aware that sore throat is one of the symptoms of oral cancer (Figure 8a). It shows that this survey helped almost $60 \%$ of the students who were included in the study, to gain knowledge over risk factors and complications of oral cancer (Figure 9a).

We have seen the association of gender and awareness of oral cancer (Figure 1b), oral cancer leading to death (Figure 2b), knowledge on oral cancer through school (Figure 3b), able to know the symptoms of oral cancer (Figure $4 b$ ), curing of oral cancer with chemotherapy and radiotherapy (Figure 5b), smoking causes oral cancer (Figure 6b), curing of oral cancer with proper diagnosis (Figure $7 \mathrm{~b}$ ), the sore throat being a symptom of oral cancer (Figure 8b), knowledge through the survey (Figure 9b)

Oral cancer was one of the least heard of cancers by the common people with only $56 \%$ of them being aware, whereas $96 \%$ had heard of skin cancer, $97 \%$ lung cancer, and $86 \%$ cervical cancer. $76 \%$ of them are aware of the link between smoking and oral cancer but only $19 \%$ were aware of its association with alcohol misuse. ${ }^{13}$ When asked without prompting what they would do if a painful mouth ulcer had lasted for more than three weeks, $61 \%$ said they would see their doctor and $27 \%$ their dentist, which is encouraging. ${ }^{14}$

The general awareness, knowledge of signs, and risk factors of oral cancer were found to be related to the qualification level with more awareness being among high school and graduates and lowest among illiterates. It was also concluded that most of the younger age groups ( $<30$ years) were comparatively more knowledgeable. ${ }^{15}$ The level of public awareness of oral cancer was $84 \%$, but only $23 \%$ for OPMD. Awareness was particularly poor in low socioeconomic groups.

The majority of the public were not aware of the symptoms of oral cancer and of OPMD. Thirty-two percent were unaware that chewing betel quid was a risk factor for these diseases, as were $65 \%$ for tobacco smoking and $81 \%$ for heavy consumption of alcohol. On the whole $76 \%$ were not aware of any of the dangers involved in the frequent use of areca nuts. ${ }^{16}$ Most of the diagnostic factors were strictly identified by the dentists, whereas less than $30 \%$ of the public were aware of these factors. Only $47 \%$ of the dentists and $27 \%$ of the public were aware that the patient is asymptomatic in the early stage of oral cancer. The knowledge about risk factors was inconsistent and showed demerits by the dentists as well as by the public. ${ }^{17}$

A statistically significant difference in general awareness of oral cancer was seen among various occupational groups. 
The attitude toward oral cancer screening was assessed and found no statistically significant results, which signifies that significant motivation for oral cancer screening is required in different occupational workplaces. ${ }^{18}$ The overwhelming majority, 93.2\% never screened for oral cancer despite their positive attitude towards it $66.4 \%$. Knowledge of risk factors related significantly with those shown a positive attitude towards oral cancer screening and those reported direct contact with health workers as a source of information. But however, females and those living in urban areas score higher than their counterparts in the knowledge of the risk factor of oral cancer. In addition, those employed $58.6 \%$ and $62.8 \%$ with correct beliefs about oral cancer reported a significant relation with positive knowledge of signs and symptoms. ${ }^{19}$

\section{CONCLUSION}

Our findings revealed that chances exist to improve knowledge, increase awareness, and develop the right practices towards oral cancer. Health workers should do more because they have a pivotal role in the early diagnosis of oral cancer by raising levels of knowledge about oral cancer. So, it can be concluded that awareness of oral cancer among school students can be a forward step in raising the levels of knowledge on complications and risk factors of Oral cancer. More research is required to study the actions of oral cancer to benefit society.

\section{Acknowledgement: None}

\section{Author Contribution}

Sushma.B - Analysis, Manuscript drafting

VishnuPriya.V - Analysis, Manuscript drafting

Karthik Ganesh Mohanraj - Analysis, Manuscript drafting

Gayathri.R - Analysis, Manuscript drafting

\section{Conflict of Interest: Nil}

Funding: None

\section{REFERENCES}

1. Mudita R, Chandrakant MP, Bhakti P. Awareness and Knowledge of Oral Cancer among Dental Practitioners of Bhopal, India - A Cross-Sectional Study. Int J Oral Dent Health 2018;4.

2. Monteiro LS, Salazar F, Pacheco J, Warnakulasuriya S. Oral cancer awareness and knowledge in the city of valongo, portugal. Int J Dent 2012;2012:376838.

3. Macpherson LMD. Raising awareness of oral cancer from a public and health professional perspective. Br Dent J 2018 Nov 9;225(9):809-14.
4. Shimpi N, Jethwani M, Bharatkumar A, Chyou P-H, Glurich I, Acharya A. Patient awareness/knowledge towards oral cancer: a cross-sectional survey. BMC Oral Health 2018 May 15;18(1):86.

5. Elango KJ, Anandkrishnan N, Suresh A, Iyer SK, RamaIyer SK, Kuriakose MA. Mouth self-examination to improve oral cancer awareness and early detection in a high-risk population. Oral Oncol 2011;47: 620-4.

6. Lowry R, Craven M. Smokers and drinkers awareness of oral cancer: a qualitative study using focus groups. Br Dent J 1999; 187: 668-70.

7. Carter LM, Ogden GR. Oral cancer awareness of undergraduate medical and dental students. BMC Med Edu 2007;7.

8. Shukri NMM, Vishnupriya V, Gayathri R, Mohan SK. Awareness in childhood obesity. Res J Pharma Tech 2016;9(10):165862.

9. Ponnulakshmi R, Shyamaladevi B, Vijayalakshmi P, Selvaraj J. In silico and in vivo analysis to identify the antidiabetic activity of beta sitosterol in adipose tissue of high fat diet and sucrose induced type-2 diabetic experimental rats. Toxicol Mech Methods 2019 May;29(4):276-90.

10. Wu F, Zhu J, Li G, Wang J, Veeraraghavan VP, Krishna Mohan $\mathrm{S}$, et al. Biologically synthesized green gold nanoparticles from Siberian ginseng induce growth-inhibitory effect on melanoma cells (B16). Artif Cells Nanomed Biotechnol 2019 Dec;47(1):3297-305.

11. Ke Y, Al Aboody MS, Alturaiki W, Alsagaby SA, Alfaiz FA, Veeraraghavan VP, et al. Photosynthesized gold nanoparticles from Catharanthus roseus induces caspase-mediated apoptosis in cervical cancer cells (HeLa). Artif Cells Nanomed Biotechnol 2019 Dec;47(1):1938-46.

12. Ma Y, Karunakaran T, Veeraraghavan VP, Mohan SK, Li S. Sesame Inhibits Cell Proliferation and Induces Apoptosis through Inhibition of STAT-3 Translocation in Thyroid Cancer Cell Lines (FTC-133). Biotech Bioproc Engi 2019;24: 646-52.

13. Warnakulasuriya K, Harris C, Scarrott D, Watt R, Gelbier S, Peters $\mathrm{T}$, et al. An alarming lack of public awareness towards oral cancer. Br Dental J 1999;187:319-22.

14. Rogers SN, Hunter R, Lowe D. Awareness of oral cancer in the Mersey region. Br J Oral Maxillofac Surg 2011 Apr;49(3):17681.

15. Agrawal M, Pandey S, Jain S, Maitin S. Oral Cancer Awareness of the General Public in Gorakhpur City, India. Asian Pac J Cancer Prev 2012;13: 5195-9.

16. Amarasinghe HK, Usgodaarachchi US, Johnson NW, Lalloo R, Warnakulasuriya S. Public awareness of oral cancer, of oral potentially malignant disorders and of their risk factors in some rural populations in Sri Lanka. Commu Dentist Oral Epidemiol 2010;38: 540-8.

17. Hertrampf K, Wenz H-J, Koller M, Wiltfang J. Comparing dentists' and the public's awareness about oral cancer in a community-based study in Northern Germany. J Cranio-Maxillofac Surg 2012;40: 28-32.

18. Amu EO. Awareness, Knowledge and Attitude to Cervical Cancer and Its Screening among Females in Somolu Local Government Area, Lagos, Nigeria. Int J Nutr Sci 2019.

19. Babiker TM, Osman KAA, Mohamed SA, Mohamed MA, Almahdi HM. Oral Cancer Awareness Among Dental Patients in Omdurman, Sudan: a cross-sectional Study. BMC Oral Health 2017;17(1):69. 


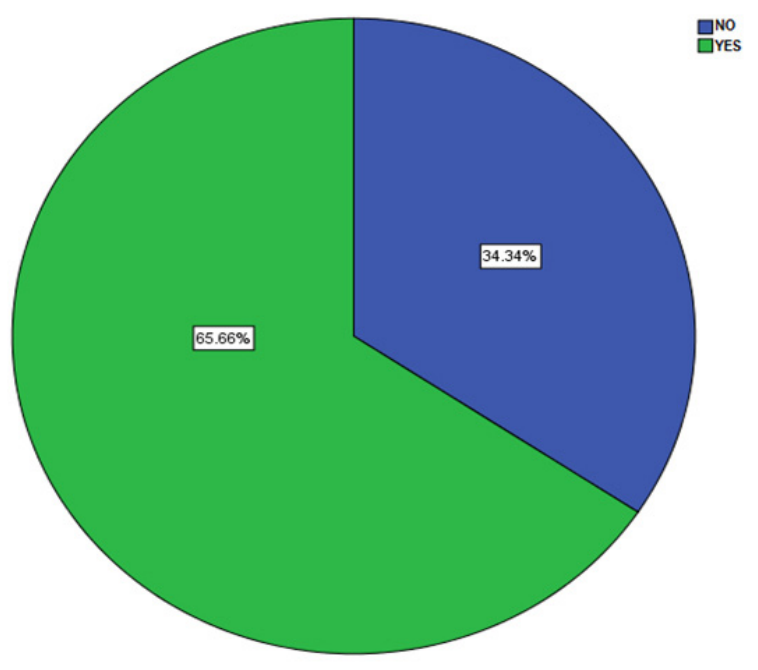

Figure 1a: The pie chart shows the percentage distribution of responses about awareness of oral cancer by school students. $66 \%$ are aware (green) and $35 \%$ are not aware of oral cancer (blue). The majority of the participants were aware of oral cancer $(66 \%)$

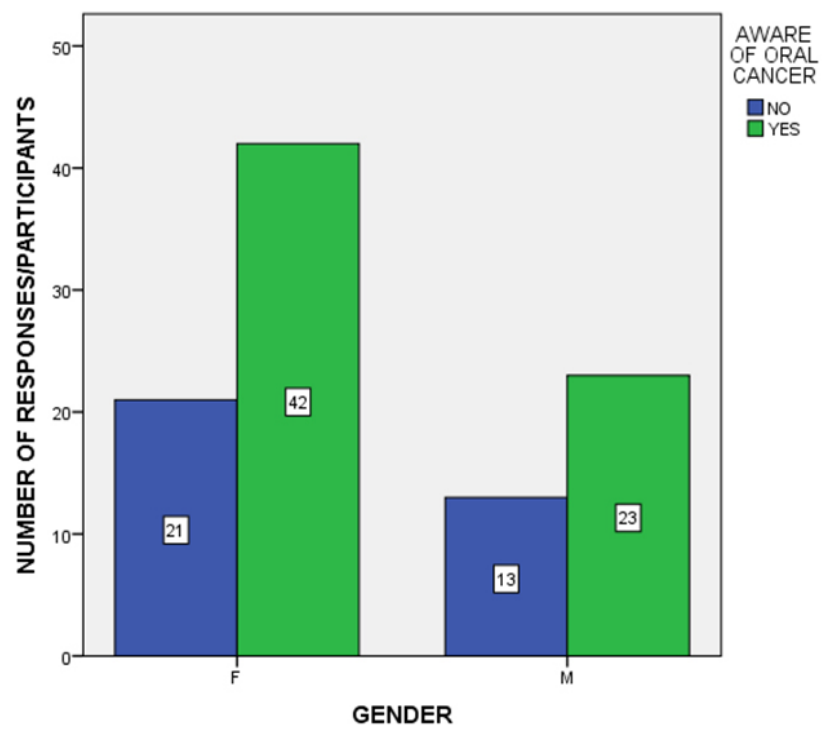

Figure 1b: Bar graph showing association between gender (Xaxis) and the number of responses about awareness on oral cancer (Y-axis). Blue denotes no and green denotes yes. Both males and females were aware of oral cancer. The majority of males and females were aware of oral cancer but on analysis there was no statistical significance between gender and awareness of oral cancer. Chi-square value $=0.078, p=0.0779$ (>0.05 statistically not significant).

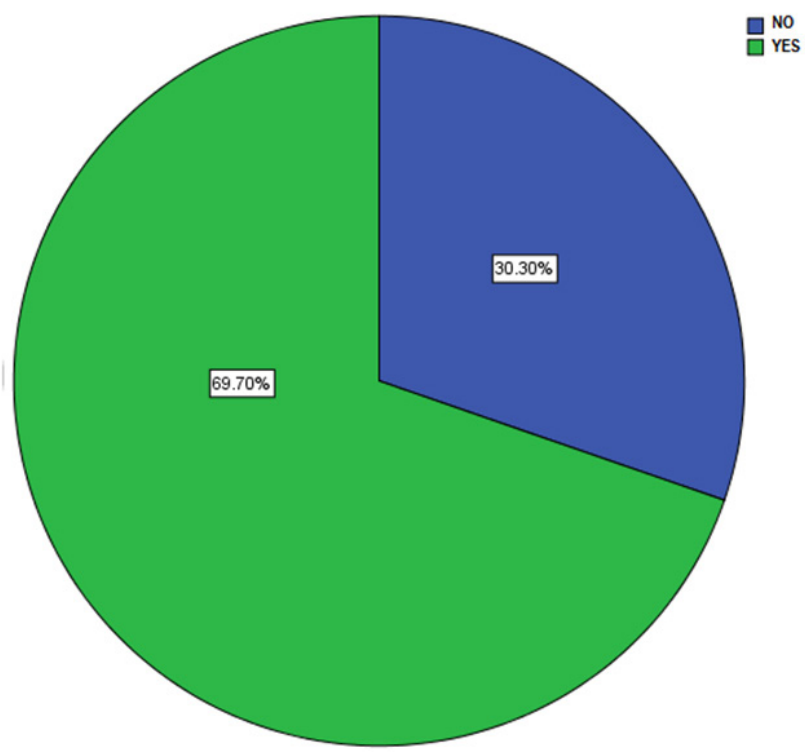

Figure 2a: The pie chart shows the percentage distribution of responses about awareness on oral cancer leading to death. $69 \%$ obey (green) and $31 \%$ do not obey that oral cancer leads to death (blue). The majority of the participants were aware that oral cancer leads to death $(69 \%)$.

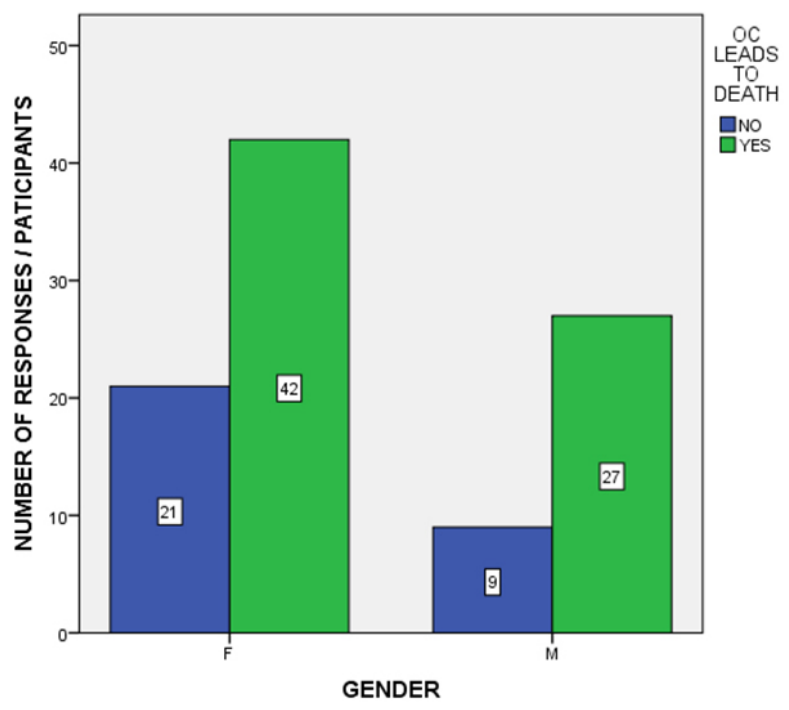

Figure 2b: Bar graph showing the association between gender (X-axis) and the number of responses about awareness on oral cancer leading to death (Y-axis). Blue denotes no and green denotes yes. Both $27 \%$ of males and $42 \%$ of females were aware that oral cancer leads to death. The majority of the males and females were aware that oral cancer leads to death but on analysis there was no statistical significance between gender and knowledge of awareness that oral cancer leads to death. Chi-square test $=0.753, p=0.385$ (>0.05 statistically not significant) 


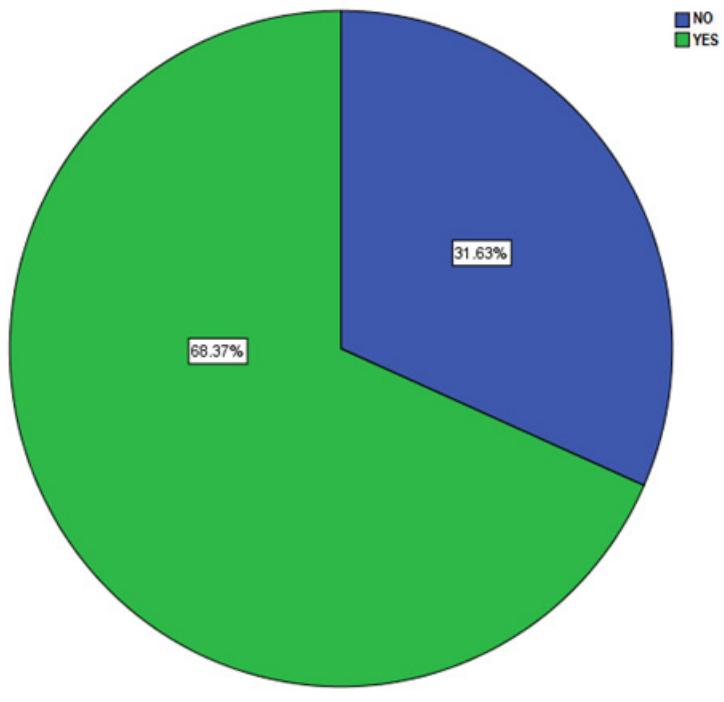

Figure 3a: The pie chart shows the percentage distribution of responses about knowledge on oral cancer in their school. $68 \%$ students were taught about oral cancer in their school (green color) and $31 \%$ students were not (blue color). It shows that most of the school students were taught about oral cancer in their school.

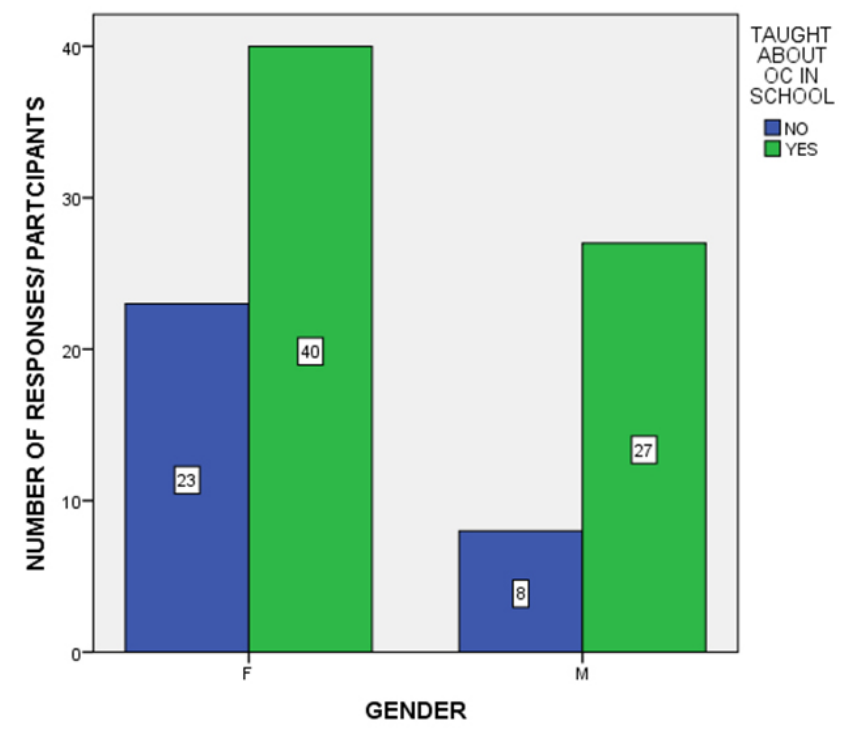

Figure 3b: Bar graph showing association between gender (X-axis) and the number of responses about knowledge on oral cancer in school (Y-axis). Blue denotes no and green denotes yes. $40 \%$ of the females and $27 \%$ of males were aware of the knowledge of oral cancer taught in school. The majority of the males and females were aware of the knowledge of oral cancer taught in school but on analysis, there was no statistical significance between gender and knowledge of oral cancer taught in school. Chi-square test $=1.939, p=0.164$ (>0.05 - statistically not significant).

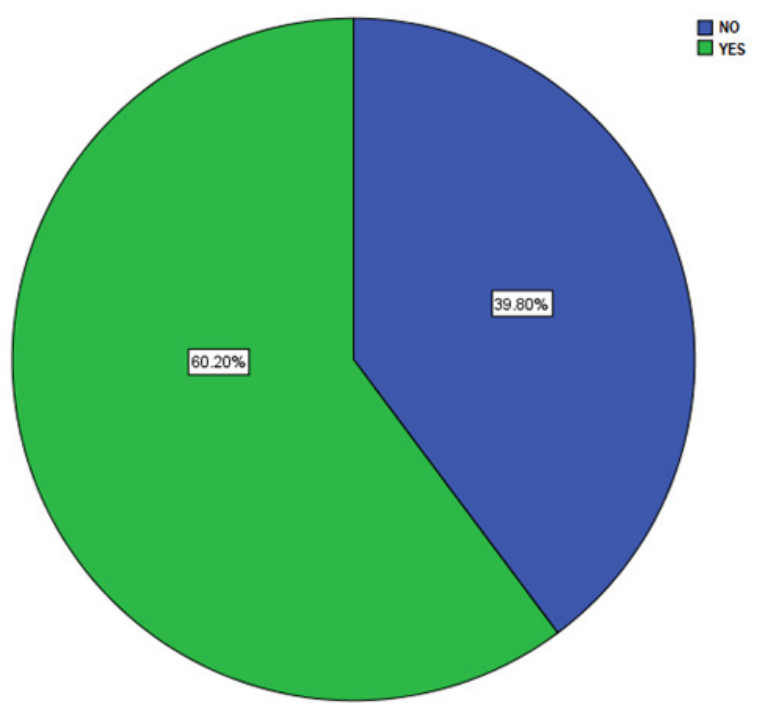

Figure 4a: The pie chart shows the percentage distribution of responses about awareness on symptoms of oral cancer. $60 \%$ students are able to know the symptom of oral cancer (green) and the other $40 \%$ students are unable to know (blue). It shows that majority of the students are able to know the symptoms of oral cancer $(60 \%)$

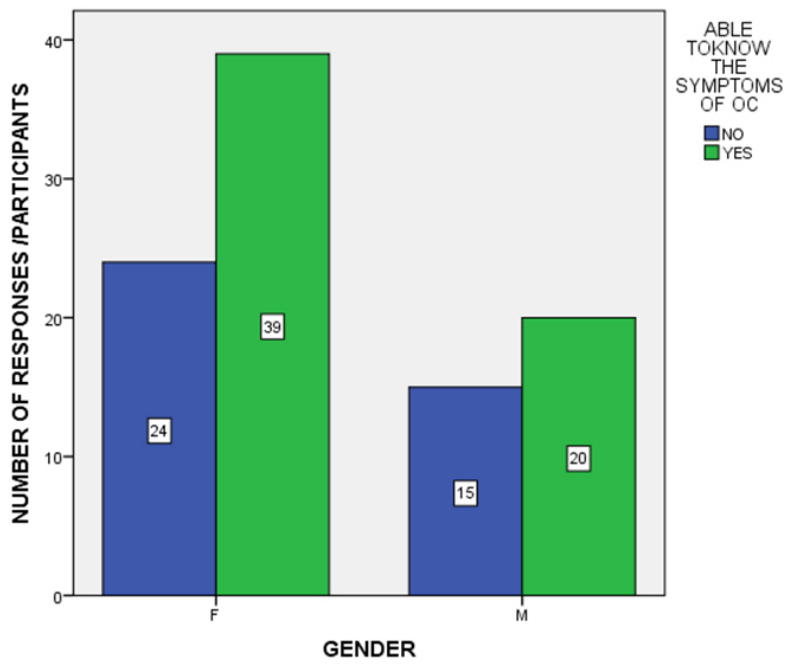

Figure 4b: Bar Graph showing the association between gender (X-axis) and the number of responses about awareness on symptoms of oral cancer (Y-axis). Blue denotes no and green denotes yes. $39 \%$ of females and $20 \%$ of males wanted to be able to know the symptoms of cancer. Most of the males and females wanted to know the symptoms of cancer but on analysis there was no statistical significance between gender and knowledge to be able to know about the symptoms of oral cancer. . Chi-square value $=0.213, p=0.644$ ( $>0.05$ considered statistically not significant). 


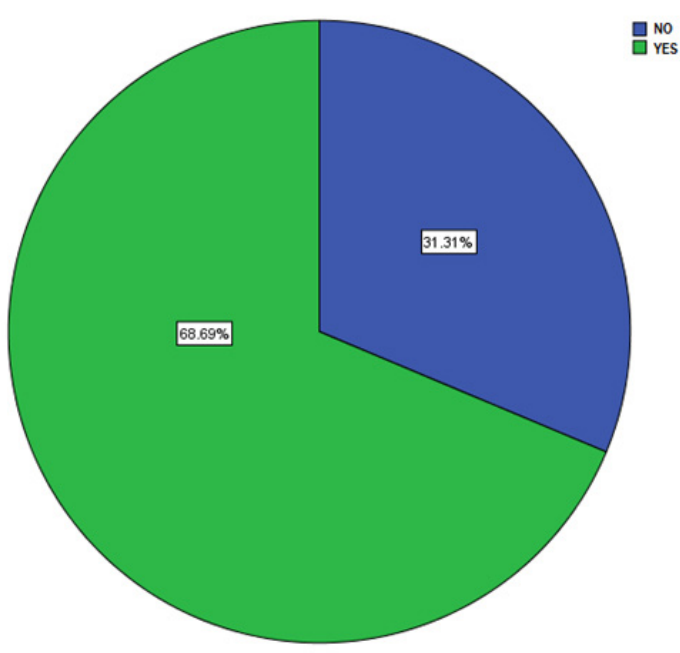

Figure 5a: The pie chart shows the percentage distribution of responses about awareness on treatment of oral cancer with chemotherapy and radiotherapy. $68 \%$ of school students are aware that oral cancer can be cured using chemotherapy and radiotherapy (green color) and the other $31 \%$ are not aware (blue color). It shows that more number of students are aware that oral cancer can be cured using chemotherapy and radiotherapy $(68 \%)$.

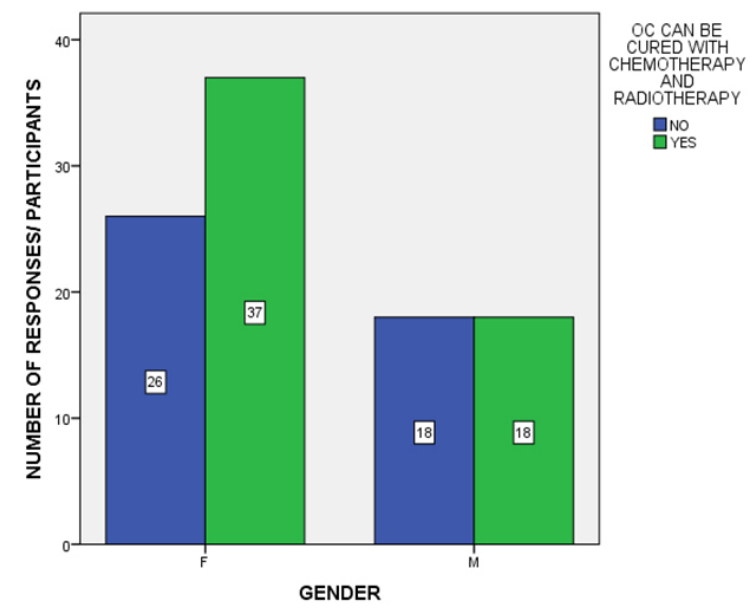

Figure 5b: Bar graph showing the association between gender (X-axis) and the responses to awareness on treatment of oral cancer with chemotherapy and radiotherapy (Y-axis). Blue denotes no and green denotes yes. $37 \%$ of the females and $18 \%$ of males were aware of the treatment to cure cancer with radiotherapy and chemotherapy. The majority of males and females were aware of the treatment to cure cancer by chemotherapy and radiotherapy but on analysis there was no statistical significance between gender and awareness of oral cancer cured through chemotherapy and radiotherapy. Chisquare value $=0.707, p=0.4(>0.05$-considered statistically not significant).

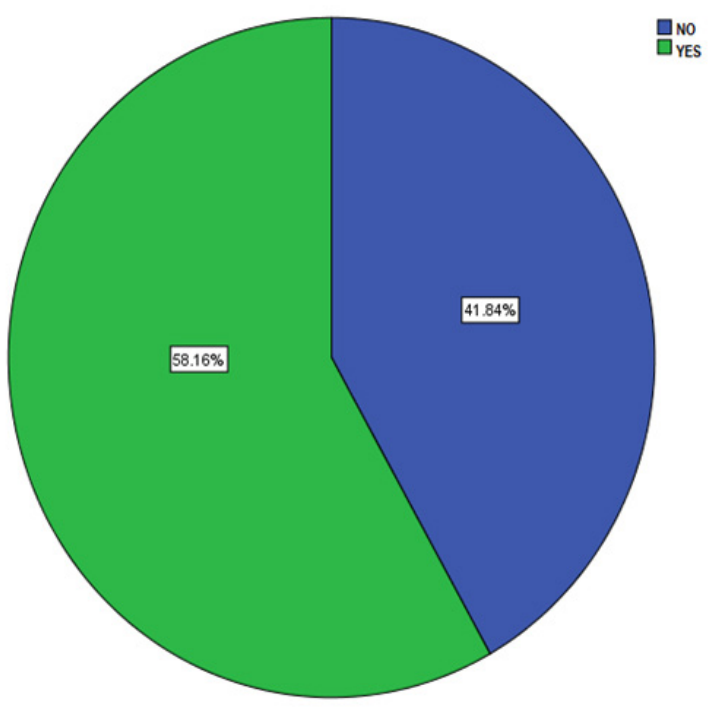

Figure 6a: The pie chart shows the percentage distribution of responses about awareness on smoking causes oral cancer. $58 \%$ of students are aware that smoking causes oral cancer (green color) and the other $41 \%$ are not aware (blue color). It shows that more students are aware that smoking causes oral cancer.

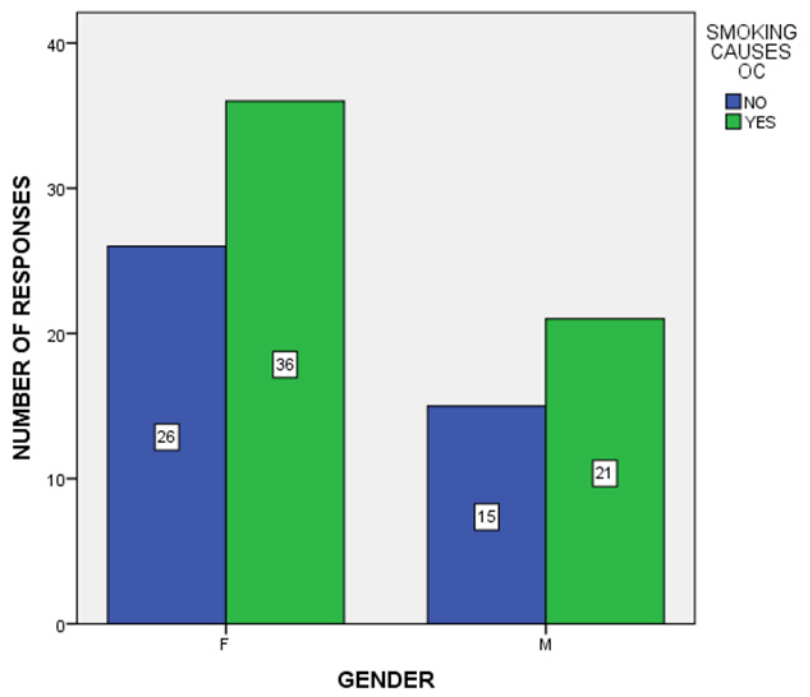

Figure 6b: Bar graph showing association between gender (X-axis) and the responses about awareness on smoking causes oral cancer (Y-axis). Blue denotes no and green denotes yes. $36 \%$ of females and $21 \%$ of males were aware that smoking causes oral cancer. The majority of the females and males were aware that smoking causes oral cancer. But in analysis there was no statistical significance between gender and awareness of smoking causes oral cancer. Chi-square test $=0.001, p=0.979(>0.05$-considered statistically not significant.) 


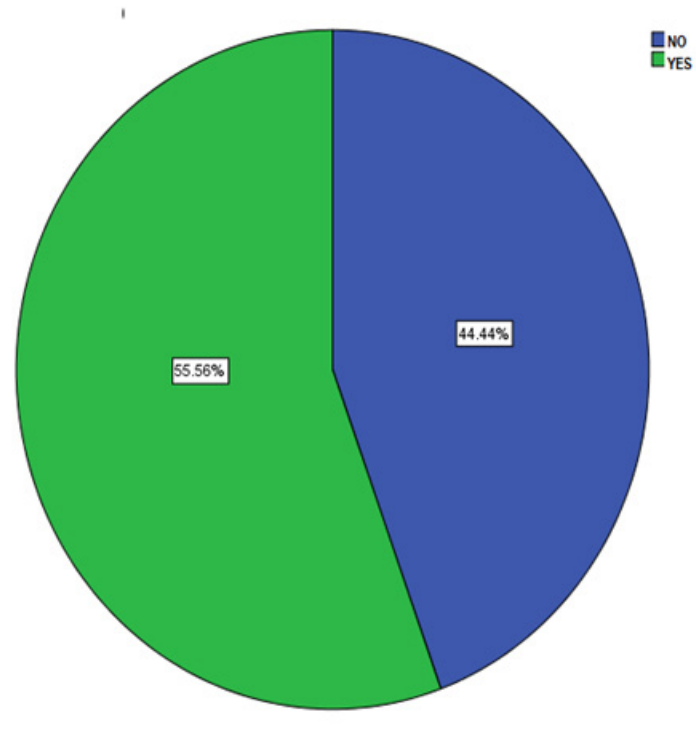

Figure 7a: The pie chart shows the percentage distribution of responses about awareness on believing that oral cancer can be cured with proper diagnosis. $55 \%$ of students obey that oral cancer can be cured through proper diagnosis (green) and $44 \%$ of students disagree (blue). The majority of the students believed that oral cancer can be cured with proper diagnosis.

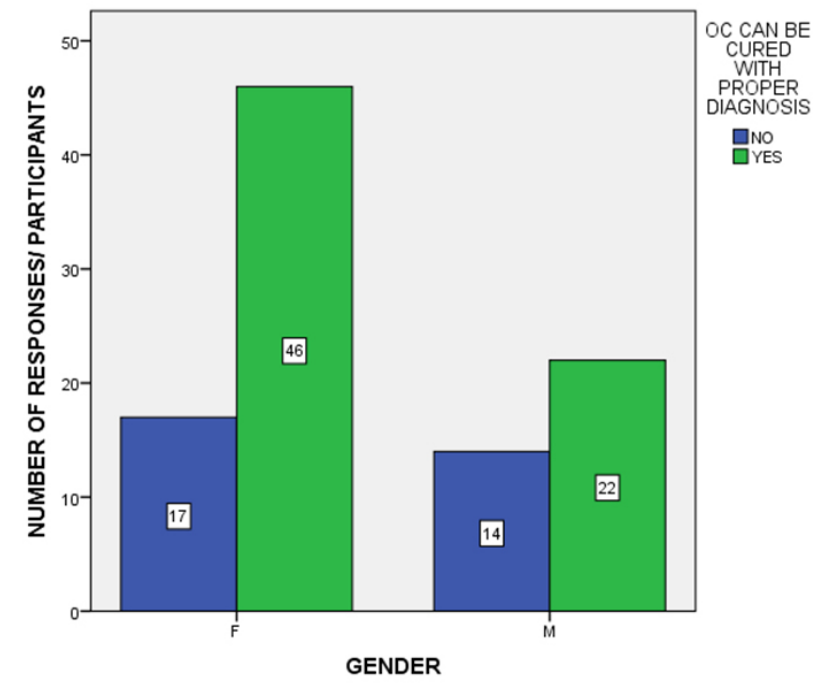

Figure 7b: Bar graph showing association between gender (X-axis) and the responses about awareness on curing of oral cancer (Y-axis). Blue denotes no and green denotes yes.46\% of females and $22 \%$ males believed that OC can be cured with proper diagnosis. The majority of males and females believed that oral cancer can be cured with proper diagnosis, the analysis is statistically not significant. Chi-square test $=1.510$, $p=0.291$ (>0.05 statistically not significant).

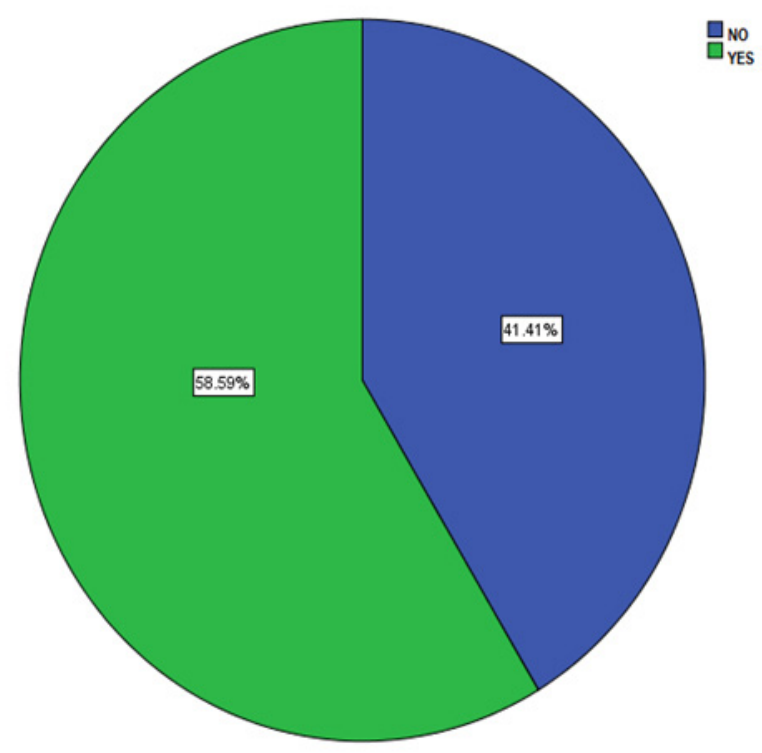

Figure 8a: The pie chart showing percentage distribution of responses about awareness on symptoms of oral cancer. $59 \%$ of students are aware that sore throat is a symptom of oral cancer (green) and the other $41 \%$ are not aware of it (blue). The majority of the school students were aware of the symptoms of oral cancer.

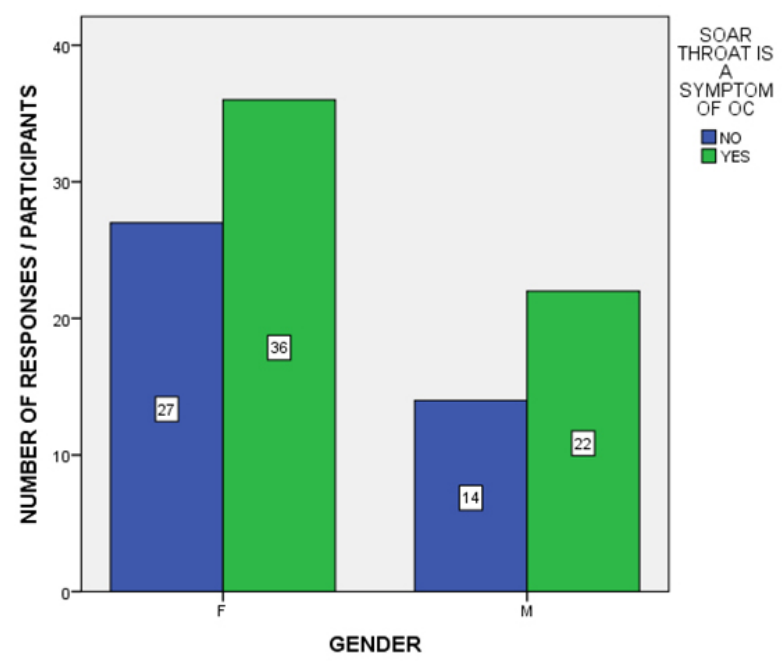

Figure 8b: Bar graph showing association between gender (X-axis) and the number of responses about awareness on symptoms of oral cancer (Y-axis). Blue denotes no and green denotes yes. $36 \%$ of females and $22 \%$ of males were aware that sore throat was a symptom of oral cancer. The majority of the female and male were aware that sore throat is a symptom of oral cancer but on analysis there was no statistical significance between gender and sore throat as a symptom of oral cancer. Chi-square test $=0.149, p=0.7$ (>0.05 -considered statistically not significant.) 


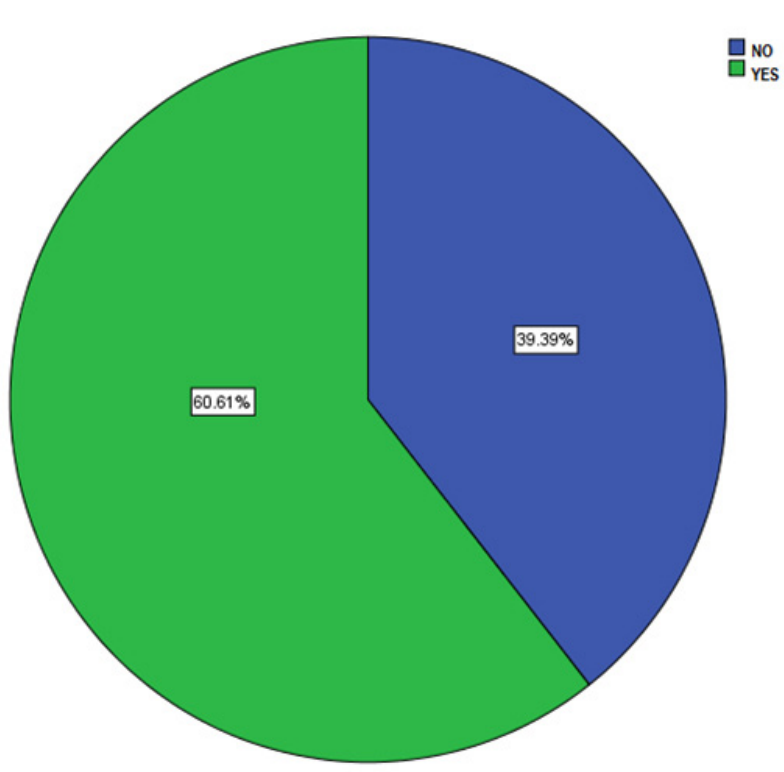

Figure 9a: The pie chart shows the percentage distribution of responses about awareness on gaining knowledge in oral cancer with this survey. Almost $60 \%$ of students were helped with this survey (green) and the other $40 \%$ are already aware of complications and risk factors of oral cancer (blue). The majority of the students have answered yes on gaining awareness on oral cancer.

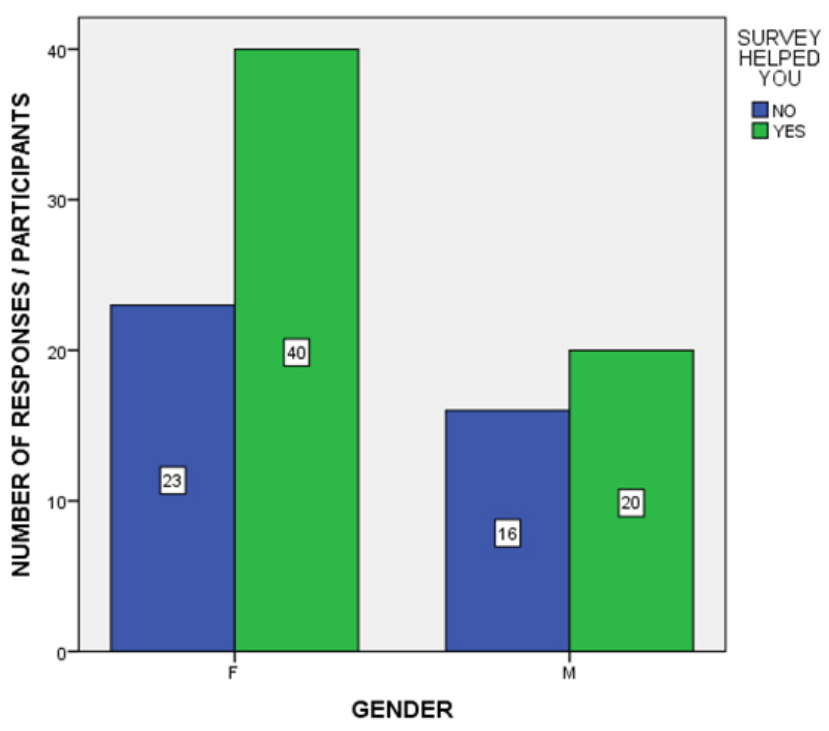

Figure 9b: Bar graph showing association between gender (X-axis) and the responses about gaining knowledge on oral cancer through the survey (Y-axis). Blue denotes no and green denotes yes. $40 \%$ of females and $20 \%$ of males found the survey helpful. The majority of the students found the survey helpful but on analysis there was no statistical significance between gender and survey. Chi-square test $=0.604, p=0.437$ (>0.05 - considered statistically not significant.) 